Hautarzt 2016 $67: 536-543$

DOI 10.1007/s00105-016-3797-z

Online publiziert: 20. Mai 2016

(c) The Author(s) 2016. This article is available at SpringerLink with Open Access

CrossMark

\author{
R. Rauschenberg ${ }^{1}$ G. Tabatabai ${ }^{2,3,4,5} \cdot$ E. G. C. Troost ${ }^{6,7,8,9,10} \cdot$ M. Garzarolli' $\cdot$ \\ S. Beissert ${ }^{1} \cdot$ F. Meier ${ }^{1}$ \\ 'Hauttumorzentrum am Universitäts Krebs-Centrum Dresden (UCC), Klinik und Poliklinik für \\ Dermatologie, Universitätsklinikum Carl-Gustav-Carus Dresden, Technische Universität Dresden, \\ Dresden, Deutschland \\ ${ }^{2}$ Interdisziplinäre Sektion Neuroonkologie, Zentrum für Neurologie und Klinik für Neurochirurgie, Hertie- \\ Institut für Klinische Hirnforschung, Universitätsklinik Tübingen, Eberhard Karls Universität Tübingen, \\ Tübingen, Deutschland \\ ${ }^{3}$ Zentrum für Neuroonkologie, Südwestdeutsches Tumorzentrum Tübingen Stuttgart, Universitätsklinik \\ Tübingen, Eberhard Karls Universität Tübingen, Tübingen, Deutschland \\ ${ }^{4}$ Zentrum für Personalisierte Medizin, Eberhard Karls Universität Tübingen, Tübingen, Deutschland \\ ${ }^{5}$ DKFZ Partnerstandort Tübingen, Deutsches Konsortium für Translationale Krebsforschung (DKTK) \\ Tübingen, Deutschland \\ ${ }^{6}$ Department of Radiation Oncology, University Hospital and Medical Faculty Carl Gustav Carus, \\ Technische Universität Dresden, Dresden, Deutschland \\ ${ }^{7}$ OncoRay - National Center for Radiation Research in Oncology, Dresden, Deutschland \\ ${ }^{8}$ Dresden and German Cancer Research Center (DKFZ), German Cancer Consortium (DKTK), Heidelberg, \\ Deutschland \\ ${ }^{9}$ Institute of Radiooncology, Helmholtz-Zentrum Dresden-Rossendorf, Dresden, Deutschland \\ ${ }^{10}$ National Center for Tumor Diseases (NCT), partner site Dresden, Dresden, Deutschland
}

\title{
Hirnmetastasen des malignen Melanoms
}

\section{Therapiebesonderheiten}

\section{Die Prognose für Melanompa- tienten mit Hirnmetastasen ist ungünstig. Etablierte lokale Be- handlungsmodalitäten stellen die Resektion, stereotaktische Radiatio oder Ganzhirnbestrahlung dar. Das Behandlungsspektrum hat sich in den letzten 5 Jahren durch die Zulas- sung wirksamer Immuntherapeutika sowie zielgerichteter Therapeutika deutlich erweitert. Hirnspezifische Resistenzmechanismen scheinen je- doch den Behandlungserfolg bei Patienten mit zerebralen Metastasen zu limitieren.}

Das maligne Melanom zählt neben Bronchial- und Mammakarzinomen zu den häufigsten Ursachen zerebraler Metastasen, die überwiegend im Spätstadium der Erkrankung auftreten [3, 13, 46]. Filiae im zentralen Nervensystem wurden bei der Autopsie von 122 Patienten mit einem histologisch gesicherten, fortgeschrittenen Melanom bei $75 \%$ der
Fälle festgestellt, wovon $46 \%$ neurologische Symptome entwickelt hatten [3]. Zu diesen Symptomen zählen insbesondere Kopfschmerzen, Verwirrung, epileptische Anfälle, (Hirnnerven-)Paresen und motorische Störungen [58]. Im Allgemeinen hat allerdings mehr als die Hälfte der Patienten zum Zeitpunkt der Erstdiagnose der zerebralen Metastasen keine Beschwerden [63].

Aufgrund der in Deutschland anhaltend hohen Prävalenz des Melanoms [22], der längeren Überlebensraten der Patienten dank innovativer effektiver Therapien (s. Abschnitt „Therapie“) sowie der weiteren Verbreitung und Optimierung der bildgebenden Verfahren (s. Abschnitt „Diagnostik“) ist von einer zunehmenden Inzidenz der Hirnmetastasen auszugehen [58]. Eine besondere Herausforderung stellt die Meningeosis carcinomatosa/neoplastica dar, d.h. die (diffuse) Ausbreitung von Tumorzellen entlang der Meningen bzw. im Subarachnoidalraum [64].
Da die Prognose für Patienten mit Hirnmetastasen mit einem medianen Überleben ohne Therapie von 2 Monaten infaust ist [12], kommt dem koordinierten klinischen Management von Hirnmetastasen eine besondere Bedeutung $\mathrm{zu}$.

Etablierte Behandlungsmodalitäten sind die neurochirurgische Resektion und - abhängig von der Metastasenanzahl und -größe - entweder die stereotaktische Radiatio oder die Ganzhirnbestrahlung.

Das Behandlungsspektrum hat sich in den letzten 5 Jahren durch die Neuzulassung von wirksamen Immuntherapien (CTLA-4- und PD-1-Antikörper) sowie zielgerichteten Therapien (BRAF [B-Form des ,rapidly accelerated fibrosarcoma"]-und MEK-Inhibitoren) zwar erweitert ([2]; s Abschnitt „Therapie“), hirnspezifische Resistenzmechanismen scheinen jedoch weiterhin Limitationen des Behandlungserfolgs darzustellen (s. Abschnitt „Besonderheiten“). 


\section{Besonderheiten}

Das maligne Melanom weist - verglichen mit anderen soliden Tumoren - eine große Anzahl an Mutationen auf [29]. Insbesondere der MAP („mitogen-activated protein“)-Kinase-Signalweg (RASRAF-MEK-ERK) ist involviert, wobei bei ca. $40-60 \%$ der kutanen Melanome eine BRAF- und bei $15-25 \%$ eine NRAS („neuroblastoma rat sarcoma virus")Mutation nachweisbar ist [30].

\section{》) Melanommetastasen nutzen den neuroprotektiven Effekt reaktiver Astrozyten}

Zerebrale Metastasen unterscheiden sich auf molekularer Ebene von anderen viszeralen Metastasen insbesondere aufgrund der vorherrschenden Aktivierung des PI3 K (Phosphatidylinositol-3-Kinase)-AKT („v-akt murine thymoma viral oncogene homolog")Überlebenssignalwegs, während sich in vergleichenden immunhistochemischen Untersuchungen bezüglich des MAPKinase-Signalwegs keine wesentlichen Unterschiede zeigen [48]. Möglicherweise spielt die Aktivierung des PI3 KAKT-Überlebenssignalweges eine Rolle bei der hirnspezifischen Therapieresistenz (s. Abschnitt „Therapie“). Zudem ist das zerebrale Mikromilieu von großer Bedeutung. Melanommetastasen nutzen den neuroprotektiven Effekt reaktiver Astrozyten. Durch die Kommunikation über "gap junctions“ und die Reduktion des Kalziums im endoplasmatischen Retikulum der Tumorzellen werden sie vor Apoptose und vor der zytotoxischen
Wirkung von Chemotherapien geschützt [33].

Eine weitere Besonderheit ist, dass beim Melanom - im Gegensatz z. B. zum Bronchialkarzinom - für die Blutversorgung der Hirnmetastasen die sog. „vessel cooption“ eine relevante Rolle spielt, d.h. die Einverleibung bestehender Gefäßstrukturen durch Tumorzellen mit resultierendem perivaskulärem Wachstum der Metastasen [24].

\section{Prognostische Faktoren}

In retrospektiven Studien korrelierte die Überlebensrate der Melanompatienten mit Hirnmetastasen im Wesentlichen mit der Anzahl zerebraler Metastasen und dem LDH (Lactatdehydrogenase)-Level im Serum [10, 63]. Für die Prognose und Therapieplanung können zusätzlich das Patientenalter, der Karnofsky-Index sowie der S100-Wert (vor der Behandlung) einbezogen werden [10].

In der multivariaten Analyse von Raizer et al. [55] waren ein hohes Alter (> 65 Jahre), das gleichzeitige Vorliegen extrazerebraler Metastasen, fokal neurologische Symptome (bei der Erstdiagnose) und 4 oder mehr Hirnmetastasen signifikant mit einer schlechteren Überlebensrate assoziiert. Von Staudt et al. [63] wurde auch die Anwesenheit ossärer Metastasen als unabhängiger prognostischer Faktor herausgestellt. Ein langes (krankheitsfreies) Intervall zwischen der Primärdiagnose und dem Zeitpunkt der Diagnose von Hirnmetastasen erwies sich in einer Serie der „Sydney Melanoma Unit“ als günstiger prognostischer Faktor [12]. Im Falle einer singulären Hirnmetastase verlängern eine stereotaktische
Radiatio oder eine neurochirurgische Resektion das Gesamtüberleben [10].

\section{Diagnostik}

Sofern der Verdacht auf eine zerebrale Metastasierung des Melanoms besteht, sollten ein Staging (Magnetresonanztomographie [MRT] des Schädels, spinales MRT und Ganzkörper-Computertomographie $[\mathrm{CT}]$ ) und eine klinisch neurologische Beurteilung (klinisch neurologische Untersuchung, neuropsychologische Testung und ggf. Lumbalpunktion) erfolgen [58].

Das variable Erscheinungsbild der Hirnmetastasen sowie Veränderungen nach einer Strahlen- oder Immuntherapie bereiten zum Teil Schwierigkeiten bei der radiologischen Diagnosestellung und Bewertung des Therapieansprechens [2]. So können sich unter einer Immuntherapie bzw. infolge einer Radionekrose Pseudoprogressionen zeigen. Von der Arbeitsgruppe „Response Assessment in Neuro-Oncology (RANO) Brain Metastases" wurden 2015 neue Kriterien zur radiologischen Beurteilung von Hirnmetastasen in klinischen Studien erstellt, um diesem Sachverhalt Rechnung zu tragen [32, 49].

\section{Therapie}

Hirnmetastasen waren bis vor Kurzem häufig ein Ausschlusskriterium für die Teilnahme an klinischen Studien. Untersuchungen zur Effektivität einer Strahlentherapie oder operativen Therapie wurden überwiegend mit heterogenen Patientenkollektiven (d.h. Patienten mit unterschiedlichen Tumorentitäten) und unter Anwendung verschiedener ra-

Hier steht eine Anzeige. 
diologischer Kriterien zur Bewertung des Therapieansprechens durchgeführt, sodass nur limitierte Daten aus randomisierten klinischen Studien zur Verfügung stehen [2]. Standardisierte Therapieregimes bzw. spezifische evidenzbasierte Leitlinien fehlen. Nicht zuletzt aufgrund der epidemiologischen Herausforderung angesichts der steigenden Inzidenz der Patienten mit Hirnmetastasen des Melanoms sind randomisierte Studien unerlässlich.

Die Therapieentscheidungbzw. -durchführung sollte interdisziplinär (im Tumorboard) bzw. multimodal unter Berücksichtigung der oben genannten prognostischen Faktoren (s. Abschnitt „Prognostische Faktoren“) erfolgen (• Abb. 1, $[2,54,58])$.

\section{Neurochirurgische Resektion}

Im Falle einer einzelnen Hirnmetastase bietet sich die Resektion an, vorausgesetzt die Läsion ist chirurgisch zugänglich und der Patient ist operationsfähig $[2,54,58]$. Insbesondere wenn symptomatische Raumforderungen verbunden mit erhöhtem Hirndruck bzw. drohender Verlegung der Liquorabflusswege vorliegen, sollte zeitnah eine operative Entlastung zur Symptomkontrolle in Erwägung gezogen werden $[54,58]$. Ein Vorteil der Metastasenresektion stellt überdies die Möglichkeit der anschließenden histologischen Untersuchung und molekularen Analyse dar.

In einer retrospektiven Untersuchung mit insgesamt 686 Patienten wurden 205 Patienten operiert [12]. Die Mehrheit der Patienten hatte lediglich eine Hirnmetastase und eine stabile oder nicht vorhandene extrazerebrale Metastasierung. Es wurden auch Patienten mit bis zu 3 symptomatischen Metastasen operiert. In dieser Studie führte die Resektion bzw. die Resektion in Kombination mit einer nachfolgenden Bestrahlung $\mathrm{zu}$ einem deutlich längeren Gesamtüberleben (8,7 Monate bzw. 8,9 Monate) als die alleinige Radiatio (3,4 Monate). Prospektive Studien zum Vergleich der stereotaktischen Radiatio mit der Resektion stehen aus.

Hautarzt 2016 - 67:536-543 DOI 10.1007/s00105-016-3797-z

(c) The Author(s) 2016

R. Rauschenberg · G. Tabatabai · E. G. C. Troost · M. Garzarolli · S. Beissert · F. Meier

Hirnmetastasen des malignen Melanoms. Therapiebesonderheiten

\section{Zusammenfassung}

Hirnmetastasen treten bei der Mehrheit der Patienten mit metastasiertem Melanom auf und sind die häufigste Todesursache. Bis vor Kurzem war die Lokaltherapie die einzige Option für die Kontrolle von Hirnmetastasen. Inzwischen stehen wirksame systemische Therapieoptionen zur Verfügung. Bei Verdacht auf Hirnmetastasen sind eine Staging-Diagnostik mit Craniale Magnetresonanztomographie (cMRT) sowie eine neurologische Untersuchung indiziert. Für die Therapieplanung sollten prognostische Faktoren wie Anzahl und Symptomatik der zerebralen Metastasen, der LDH- und S100-Wert im Serum, die extrazerebrale Metastasierung sowie der ECOG (Eastern Cooperative Oncology Group)-Status einbezogen werden. Die Therapieentscheidung bzw. -durchführung sollte interdisziplinär bzw. multimodal erfolgen. Bei singulären Hirnmetastasen sind die neurochirurgische Resektion und die stereotaktische Radiatio etabliert. Das Behandlungsspektrum hat sich durch die Neuzulassung von wirksamen Immuntherapien (CTLA-4- und PD-1-Antikörper) sowie zielgerichteten Therapien (BRAFund MEK-Inhibitoren) erheblich erweitert. Die palliative Ganzhirnradiatio wird bei multiplen symptomatischen Hirnmetastasen eingesetzt, wobei bisher kein signifikanter Vorteil für das Gesamtüberleben gezeigt werden konnte. Bei erhöhtem intrakraniellem Druck und epileptischen Anfällen sind Kortikosteroide und Antikonvulsiva indiziert. In aktuellen klinischen Studien werden für Melanompatienten mit Hirnmetastasen neue Therapieoptionen wie PD-1-Antikörper, Ipilimumab plus Nivolumab, BRAF-Inhibitoren plus MEK-Inhibitoren sowie stereotaktische Radiatio in Kombination mit Immuntherapie bzw. zielgerichteter Therapie untersucht.

Schlüsselwörter

Resektion · Radiatio · Systemtherapie · BRAFInhibitor $\cdot$ Immuntherapie

\section{Melanoma brain metastases. Treatment options}

\section{Abstract}

The majority of patients with metastatic melanoma will develop brain metastases, which are the most common cause of death. Until recently, local therapies (e. g., neurosurgery, radiotherapy) were the only options for brain metastases; however, effective systemic treatment options are now available. Upon suspicion of brain metastases, diagnostic staging with brain MRI and a neurological investigation are indicated. Prognostic factors such as number of cerebral metastases and symptoms, serum lactate dehydrogenase and S-100 levels, extracerebral metastases, and ECOG status are considered during therapeutic planning. Treatment planning and therapeutic interventions should be based on an interdisciplinary and multimodal approach. Established treatments for singular brain metastases are neurosurgical resection and stereotactic radiotherapy, which can prolong survival. In patients with asymptomatic
BRAF V600E-mutant brain metastases, the BRAF inhibitors dabrafenib, vemurafenib, and immunotherapy with ipilimumab are used. In the case of multiple symptomatic brain metastases, palliative whole-brain radiotherapy is used for treatment, although it has failed to show an overall survival benefit. Increased intracranial pressure and epileptic seizures are addressed with corticosteroids and anticonvulsants. Current clinical studies for melanoma patients with brain metastases are investigating new treatment options such as PD-1 antibodies, combined ipilimumab and nivolumab, combined BRAF inhibitors and MEK inhibitors, and stereotactic radiation in combination with immunotherapy or targeted therapy.

Keywords Surgery - Radiotherapy · Systemic therapy · BRAF inhibitors · Immunotherapy 


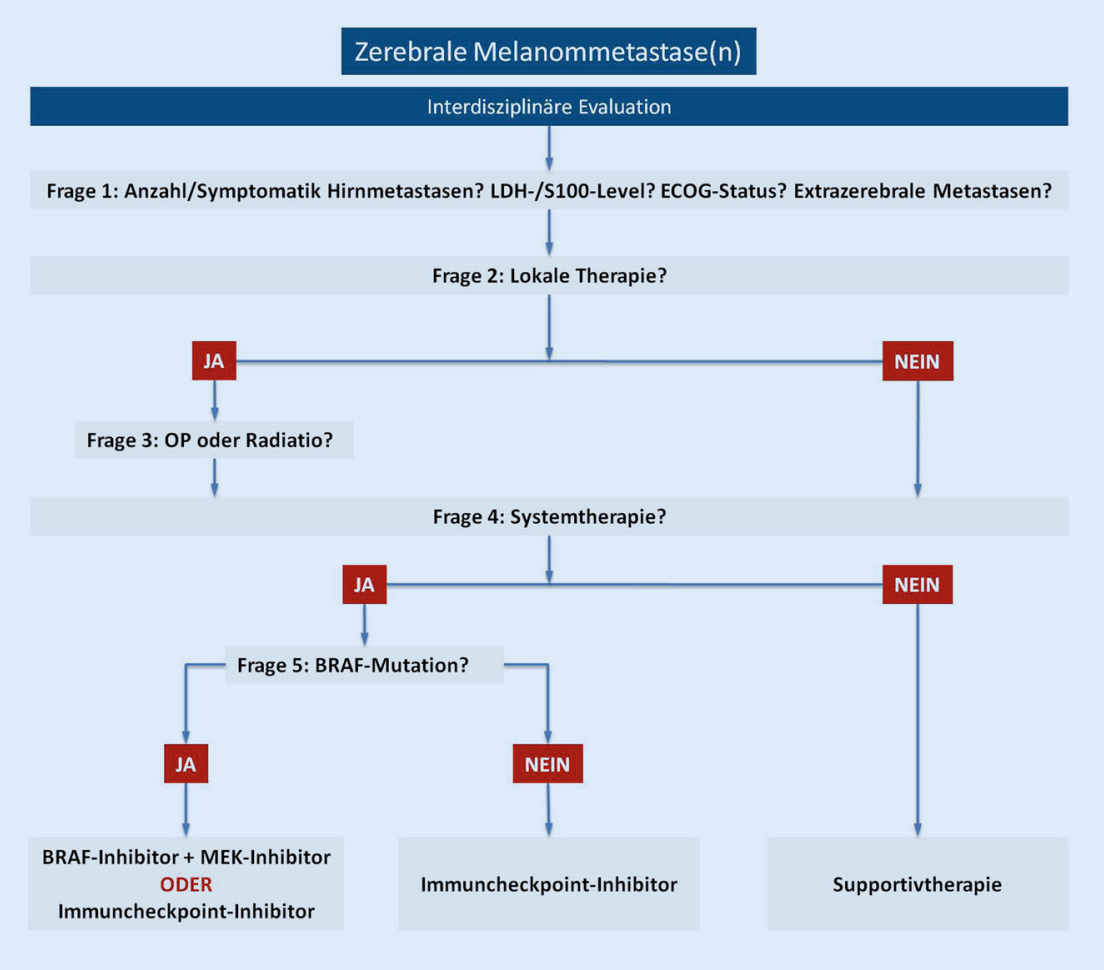

Abb. 1 ॥ ECOG Eastern Cooperative Oncology Group, OP Operation Behandlungsalgorithmus für Patienten mit zerebralen Melanommetastasen

\section{Stereotaktische Radiatio}

Alternativ zur Resektion kann Patienten mit 3 bis 4 Metastasen (ggf. $\leq 10$ Metastasen) und einer Größe von 0,5-4 cm eine minimalinvasive, stereotaktische Radiatio in 1 bis 6 Fraktionen angeboten werden $[2,38,58,66]$. Unter Verwendung eines Linearbeschleunigers, Gamma-Knifes oder Cyberknifes und modernen Bestrahlungstechniken mit steilem Dosisabfall werden die umgebenden Strukturen (Hirngewebe, Hirnstamm usw.) geschont.

In einer retrospektiven Studie konnte 2006 bei 221 stereotaktisch behandelten Melanomhirnmetastasen eine lokale Kontrollrate von $84 \%$ bei geringer Toxizität (ca. $5 \%$ ) erzielt werden [16]. Das mediane Gesamtüberleben der Patienten in dieser Studie lag bei 5,1 Monaten.

Risiken stellen akute neurotoxische Effekte wie Kopfschmerzen, Übelkeit, Erbrechen und epileptische Anfälle aufgrund von Ödem und Hämorrhagie dar. Zudem sind bei einer Bestrahlung im Langzeitverlauf Radionekrosen oder Leukenzephalopathien möglich $[4,6]$. Bei Verzicht auf eine Ganzhirnbestrah- lung und die damit assoziierten Nebenwirkungen können sich des Weiteren neue metastatische Läsionen außerhalb des Bestrahlungsfeldes entwickeln.

In Studien werden unterschiedliche mediane Dosierungen (zwischen 18 und 25 Gray) verwendet bzw. unterschiedliche Tumorvolumina bestrahlt (zwischen 0,6 und $5,6 \mathrm{~cm}^{3}$ ) [6], sodass keine detaillierten Empfehlungen gegeben werden können, die speziell für die stereotaktische Radiatio zerebraler Melanommetastasen geeignet sind.

Ein Paradigmenwechsel hin zur sog. „isotoxic dose prescription“ (IDP) basierend auf spezifischen Toleranzlevels der Risikoorgane wird insbesondere für große Metastasen und solche in funktionskritischen Arealen gefordert [68].

\section{Ganzhirnradiatio}

Die Radiation Therapy Oncology Group (RTOG) empfahl 1997 basierend auf einer Recursive Partitioning Analysis (RPA) für Studien zur Radiatio von Hirnmetastasen die Zuordnung von Patienten in 3 prognostische Gruppen [14]:
- RPA-Klasse 1: Patienten mit einem Karnofsky-Index $\geq 70,<65$ Jahre alt, mit einer kontrollierten Hirntumorerkrankung und fehlenden extrazerebralen Metastasen,

- RPA-Klasse 3: Patienten mit einem Karnofsky Performance Status (KPS) $<70$,

- RPA-Klasse 2: alle anderen Patienten (mit einem Karnofsky-Index $\geq 70$ ).

Die Ganzhirnradiatio stellt für Patienten mit multiplen symptomatischen Hirnmetastasen (insbesondere der RPA-Klasse 2) eine palliative Behandlungsoption dar. Hierbei werden Gesamtdosen von 20 oder 30 Gray in 5 bis 10 Fraktionen über 1 bis 2 Wochen appliziert. Bisher wurde für die Ganzhirnradiatio kein statistisch signifikanter Vorteil für das Gesamtüberleben gezeigt, allerdings wird wahrscheinlich der symptomatische zerebrale Progress verzögert [58].

\section{》) Die Ganzhirnradiatio ist eine palliative Behandlungsoption}

Demgegenüber müssen akute und langfristige neurotoxische Effekte der Ganzhirnradiatio bei der Indikationsstellung berücksichtigt werden, z. B. Fatigue, Alopezie, Xerostomie und kognitive Störungen [18]. Zur Prävention dieser letztgenannten Nebenwirkungen wird die Hippocampus-schonende Ganzhirnbestrahlung in verschiedenen klinischen Studien untersucht [17, 18].

Der Stellenwert der adjuvanten Ganzhirnradiatio ist nicht geklärt [54]. In prospektiven Studien, in die Patienten mit unterschiedlichen Tumorentitäten eingeschlossen wurden, konnte für eine adjuvante Ganzhirnradiatio weder nach der Operation einer zerebralen Metastase noch nach der stereotaktischen Radiatio von bis zu 4 zerebralen Metastasen ein Überlebensvorteil nachgewiesen werden [4, 50, 62]. Lediglich die Kontrolle lokaler und entfernter Hirnmetastasenrezidive war nach einer adjuvanten Ganzhirnradiatio verbessert. In einer retrospektiven Analyse wurde allerdings eine Verlängerung des Gesamtüberlebens für Melanompatienten mit Hirnmetastasen beobachtet, die eine adjuvante Ganzhirn- 
bestrahlung erhielten ( 7 vs. 9 Monate; $p=0,0458$ ) [10].

Zur Frage der adjuvanten Ganzhirnradiatio nach Operation oder stereotaktischer Radiatio von Melanomhirnmetastasen wird aktuell von der Australia \& New Zealand Melanoma Trials Group (ANZMTG) eine prospektive Studie durchgeführt (TROG 08.05 [WBRT Mel]).

\section{Supportivtherapie}

Patienten mit einer symptomatischen zerebralen Metastasierung bedürfen einer adäquaten Supportivtherapie [54, 58]. Die wichtigsten Behandlungsziele sind hier die Kontrolle des erhöhten intrakraniellen Drucks durch Kortikosteroide (z.B. Dexamethason p.o. oder i.v.) und die Kontrolle epileptischer Anfälle durch Antikonvulsiva (z. B. Levetiracetam p.o.). Kortikosteroide sollten „so viel wie nötig und so wenig wie möglich" eingesetzt, bei Bedarf gesteigert und nach erfolgreicher Therapie ausschleichend abgesetzt werden. Antikonvulsiva werden häufig für die gesamte Überlebenszeit benötigt. Bei Kontrolle der Hirnmetastasen durch die Therapie und Anfallsfreiheit über mehrere Monate kann das Antikonvulsivum ausschleichend abgesetzt werden. Bei akuter Symptomatik durch Hirnmetastasen sollten insbesondere die neurochirurgischen und radiochirurgischen Therapieoptionen geprüft werden.

\section{Systemtherapien}

\section{Chemotherapie}

Chemotherapien spielen für die Behandlung von Melanomhirnmetastasen eine untergeordnete Rolle. Vor 2011 (vor der Zulassung von Ipilimumab) galt die Behandlung mit dem Alkylans Dacarbazin (DTIC) als Standardtherapie für Patienten mit nicht operablen Melanommetastasen [56]. Alternativ wurde das liquorgängige Derivat Temozolomid oder der Nitrosoharnstoff Fotemustin eingesetzt - ohne jeweils zu einer signifikanten Verlängerung des Gesamtüberlebens $\mathrm{zu}$ führen $[5,41,51]$. In einer Phase3-Studie wurden 76 Melanompatienten mit Hirnmetastasen mit Fotemustin al- leine $\left(100 \mathrm{mg} / \mathrm{m}^{2}\right.$ an Tag $1,8,15$ und nach 5-wöchiger Pause alle 3 Wochen bis zum Progress) oder mit einer begleitenden Ganzhirnbestrahlung (2,5 Gy/Tag an Tag 1 bis 5 für 3 Wochen) behandelt [44]. Zwar konnte die Zeit bis zum zerebralen Progress durch die Kombination verlängert werden. Ein signifikanter Unterschied hinsichtlich der zerebralen Ansprech- und Kontrollraten sowie des Gesamtüberlebens war jedoch nicht nachweisbar. Ebenso erzielte die Kombination Temozolomid plus Ganzhirnradiatio keinen Überlebensvorteil [59].

\section{Zielgerichtete Therapie}

Die Serin-Threonin-Kinase BRAF ist ein integraler Bestandteil des MAPKinase-Signaltransduktionswegs. Bei ca. 40-60\% der kutanen Melanome liegt eine aktivierende BRAF-Mutation vor, die zu unkontrolliertem Tumorzellwachstum führt [7].

In Deutschland sind für die Systemtherapie des metastasierten Melanoms die BRAF-Inhibitoren Vemurafenib und Dabrafenib zugelassen. Beide BRAFInhibitoren konnten auch eine Wirksamkeit bei Hirnmetastasen demonstrieren. In einer Pilotstudie wurden 24 Melanompatienten mit vortherapierten symptomatischen Hirnmetastasen und einer nachgewiesenen BRAF-V600Mutation mit Vemurafenib 960 mg 2-mal täglich oral behandelt [9]. Bei 16\% der Patienten wurde eine partielle Remission der Hirnmetastasen beobachtet; $68 \%$ der Patienten zeigten eine Stabilisierung der Hirnmetastasen. Das mediane Gesamtüberleben betrug 5,3 Monate. In dieser Pilotstudie unterschied sich das Sicherheitsprofil nicht wesentlich von dem vorausgegangener Studien mit Vemurafenib (vgl. [56].). In einer Phase-2-Studie erzielte Vemurafenib bei BRAF-V600mutierten Melanompatienten mit asymptomatischen und symptomatischen Hirnmetastasen nach bzw. ohne Vortherapie eine intrakranielle Ansprechrate von 20 bzw. $18 \%$, ein medianes progressionsfreies Überleben (PFS) von 4,0 bzw. 3,7 Monaten und ein Gesamtüberleben (OS) von > 6 Monaten [23].

In einer weiteren Phase-2-Studie (BREAK-MB) erhielten 172 BRAFV600-mutierte Melanompatienten mit mindestens einer 5-40 mm messenden, asymptomatischen, unbehandelten oder lokal vortherapierten Hirnmetastase den BRAF-Inhibitor Dabrafenib $150 \mathrm{mg}$ 2-mal täglich oral [36]. Patienten mit BRAF-V600E-Mutation zeigten ohne bzw. nach Vortherapie eine intrakranielle Ansprechrate („partial response“ [PR] plus „complete response“ [CR]) von 39,2 bzw. $30,8 \%$, wobei die intrakranielle Krankheitskontrollrate (CR + $\mathrm{PR}+$ „stable disease“ [SD]) 50 bzw. $46,7 \%$ betrug. Zudem ergaben sich ein medianes PFS von 16,1 bzw. 16,6 Wochen und ein medianes OS von 33,1 bzw. 31,4 Wochen. Schwerwiegende Nebenwirkungen traten bei $30 \%$ der Patienten auf, vorwiegend Pyrexie, Plattenepithelkarzinome der Haut und intrakranielle Blutungen. Lediglich $2 \%$ der Patienten brachen die Behandlung aufgrund von Nebenwirkungen ab.

Es ist bisher unklar, ob Dabrafenib bei Hirnmetastasen wirksamer ist als Vemurafenib. Beide BRAF-Inhibitoren sind Substrate für Effluxtransporter, die bedeutsam für den Rücktransport von Stoffen aus dem Gehirn in den Blutkreislauf sind $[42,43]$.

Insgesamt scheinen BRAF-Inhibitoren bei Hirnmetastasen weniger effektiv zu sein als bei extrazerebralen Metastasen [53]. In einer retrospektiven Analyse der Daten von 86 Melanompatienten, die mit Vemurafenib behandelt worden waren, wurde bei $59 \%$ der Patienten ein unterschiedliches Therapieansprechen von zerebralen und extrazerebralen Metastasen mit anhaltendem Ansprechen der extrazerebralen Metastasen bei gleichzeitig neu aufgetretenen Hirnmetastasen beobachtet.

Eine Limitation der Monotherapie mit einem BRAF-Inhibitor stellt die Resistenzentwicklung dar, die bei der Mehrheit der Patienten im Behandlungsverlauf auftritt. Die meisten bisher beschriebenen Resistenzmechanismen führen zu einer Reaktivierung des MAP-Kinase-Signalwegs [47, 61]. Extrazerebral ist die Überlegenheit der Kombination von einem BRAF- und einem MEK-Inhibitor im Vergleich zu Dabrafenib oder Vemurafenib alleine gut belegt [28, 35], sodass in Deutschland die Kombinationstherapie mit Dabrafenib plus Trameti- 
nib bzw. mit Vemurafenib plus Cobimetinib für die Systemtherapie des metastasierten Melanoms zugelassen ist. Aktuell werden klinische Studien mit Dabrafenib plus Trametinib bzw. mit Vemurafenib plus Cobimetinib für Melanompatienten mit Hirnmetastasen durchgeführt (NCT02039947 bzw. NCT02230306).

\section{》) Die Monotherapie mit einem BRAF-Inhibitor ist aufgrund Resistenzentwicklung limitiert}

BRAF-Inhibitoren, insbesondere Vemurafenib, können signifikante radiosensitivierende Effekte auf gesundes Gewebe haben [67]. Radiosensitivierung bzw. Radiation-Recall-Reaktionen wurden bei Patienten beobachtet, die vor, während oder nach der Therapie mit Vemurafenib bestrahlt wurden. Am häufigsten war die Haut betroffen - sehr selten der Ösophagus, die Leber, das Rektum, die Lunge und die Harnblase. Rompoti et al. [57] beobachteten infolge der Kombination einer Ganzhirnradiatio mit Vemurafenib bei 3 Patienten eine Radiodermatitis (bis CTCAE [Common Terminology Criteria for Adverse Events] Grad 2) am Capillitium. Jedoch stimmt die große Mehrheit retrospektiver Analysen darin überein, dass die Kombination einer stereotaktischen Radiatio mit einem BRAF-Inhibitor die Toxizität gegenüber einer stereotaktischen Radiatio oder einem BRAFInhibitor alleine nicht erhöht $[1,20,45$, 57, 67]. Diese Beobachtung wird darauf zurückgeführt, dass die stereotaktische Radiatio einer Hirnmetastase das umliegende gesunde Gewebe weitestgehend schont, während bei einer Ganzhirnradiatio die Haut ca. $70 \%$ der verschriebenen Dosis erhält [57].

Eine Radionekrose wurde bei einem Patienten berichtet, der eine stereotaktische Radiatio unter der Therapie mit Vemurafenib erhielt, und bei 2 Patienten, die 1 bis 2 Wochen nach einer stereotaktischen Radiatio mit Vemurafenib behandelt wurden [31, 52]. Retrospektiv wird allerdings die Inzidenz von Radionekrosen nach einer stereotaktischen Bestrahlung alleine mit 5,2\% nach 6 Monaten und $17,2 \%$ nach 12 Monaten angegeben [27]. Zusammengefasst sprechen die bis- her vorliegenden retrospektiven Daten dafür, dass die Kombination einer stereotaktischen Radiatio mit einem BRAFInhibitor nicht die Radiotoxizität erhöht, die lokale Kontrolle der Hirnmetastasen verbessert und das Überleben verlängert $[15,37]$. Jedoch muss diese klinisch relevante Frage im Rahmen prospektiver Studien untersucht werden.

Die Aktivierung des PI3 K-AKTÜberlebenssignalweges ist ein charakteristisches Merkmal von Melanomhirnmetastasen (s. Abschnitt „Besonderheiten"). Aktuell wird in einer Phase-2Studie der PI3 K-Inhibitor Buparlisib bei Melanompatienten mit Hirnmetastasen nach der Vortherapie mit einem BRAFInhibitor bzw. einem ImmuncheckpointInhibitor untersucht (NCT02452294).

\section{Immuntherapie}

Der CTLA-4-Antikörper Ipilimumab, der das zytotoxische T-Lymphozytenassoziierte Antigen auf der T-Zelle blockiert und somit die Antitumorimmunität augmentiert, erzielte als erstes Systemtherapeutikum eine signifikante Verlängerung des Gesamtüberlebens bei Patienten mit metastasiertem $\mathrm{Me}$ lanom [21] und ist in Deutschland in einer Dosierung von $3 \mathrm{mg} / \mathrm{kg}$ i. v. alle 3 Wochen für 4 Dosen zugelassen. Aktivierte T-Zellen sind in der Lage, die Blut-Hirn-Schranke zu passieren [65]. In einer Phase-2-Studie (CA184-042) wurden 72 Patienten mit asymptomatischen oder symptomatischen steroidpflichtigen Melanomhirnmetastasen mit Ipilimumab 10 mg/kg 4-mal im Abstand von 3 Wochen therapiert [39]. Im Falle einer Remission oder Stabilisierung der Metastasen wurde die Therapie mit Ipilimumab alle 12 Wochen fortgeführt. Die asymptomatischen Patienten zeigten eine intrakranielle Krankheitskontrollrate (CR, PR, SD) von $24 \%$, ein medianes Gesamtüberleben von 7 Monaten und eine 2-Jahres-Überlebensrate von $26 \%$. Im Vergleich dazu betrugen die intrakranielle Krankheitskontrollrate bei Symptomatik und Steroideinnahme $10 \%$, das Gesamtüberleben 3,7 Monate und die 1-Jahres-Überlebensrate $10 \%$. Zusammengefasst scheint Ipilimumab am ehesten bei asymptomatischen Hirnmetastasen wirksam zu sein. Die häufigsten (immunvermittelten) Nebenwirkungen waren Fatigue, Diarrhö, Übelkeit, Kopfschmerzen, Exantheme und Juckreiz. Ein Patient verstarb an den Folgen einer Kolitis. Eine frühzeitige Diagnose der zum Teil lebensbedrohlichen Nebenwirkungen und deren adäquate Behandlung ggf. unter Einsatz von systemischen Glukokortikosteroiden und anderen Immunsuppressiva sind erforderlich [11].

\section{》) Ipilimumab scheint am ehesten bei asymptomatischen Hirnmetastasen wirksam zu sein}

Follow-up-Daten einer Phase-2-Studie (NIBIT-M1), in der insgesamt 86 Patienten mit einem metastasierten Melanom eine Kombinationstherapie mit Ipilimu$\mathrm{mab}(10 \mathrm{mg} / \mathrm{kg}$ 4-mal im Abstand von 3 Wochen) und Fotemustin $\left(100 \mathrm{mg} / \mathrm{m}^{2}\right.$ 3-mal im Abstand von 1 Woche und alle 3 Wochen in Woche 9 bis 24) erhielten, zeigten für 20 Patienten mit asymptomatischen Hirnmetastasen ein medianes Gesamtüberleben von 12,7 Monaten und eine 3-Jahres-Überlebensrate von $27,8 \%$ bei allerdings erheblicher Toxizität (Thrombozytopenie, Neutropenie, Transaminasenerhöhung) [8].

Seit 2015 sind in Deutschland die PD („programmed death“)-1-Antikörper Nivolumab und Pembrolizumab für die Therapie des fortgeschrittenen (nichtresezierbaren oder metastasierten) Melanoms zugelassen. Frühe Daten einer Phase-2-Studie mit Pembrolizumab $10 \mathrm{mg} / \mathrm{kg}$ i.v. alle 2 Wochen bei 18 Melanompatienten mit mindestens einer asymptomatischen 5-20 mm messenden Hirnmetastase deuten auf eine intrakranielle Wirksamkeit von Pembrolizumab hin mit einer Ansprechrate (PR) von 22-29\% und einer 6-Monats-Überlebensrate von $67 \%$ bei gut kontrollierbaren Nebenwirkungen [25].

In Zusammenschau der bisher vorliegenden retrospektiven Daten scheint eine Kombinationstherapie von $\mathrm{Me}$ lanomhirnmetastasen mit Ipilimumab und einer Strahlentherapie nicht mit einer erhöhten Toxizität einherzugehen, wobei bisher unklar ist, ob diese Kombinationsstrategie eine Verlängerung des Überlebens erzielen kann [19, 26, 40,60]. 
In einer retrospektiven Analyse wurde bei 39 Patienten mit metastasiertem Melanom die Sicherheit und Wirksamkeit einer Kombinationstherapie mit PD-1-Antikörpern und Radiatio untersucht [34]. Diese Kombinationsstrategie schien nicht mit einer erhöhten Toxizität einherzugehen. Sieben Patienten erhielten eine Ganzhirnradiatio und 6 Patienten eine stereotaktische Radiatio der Hirnmetastasen, wobei bei 2 Patienten, die Ipilimumab, Pembrolizumab und eine Ganzhirnradiatio erhielten, ein Hirnödem bzw. akute kognitive Defizite auftraten.

\section{Konklusionen und Perspektiven}

Aktuell stehen für Patienten mit metastasiertem Melanom und Hirnmetastasen mehrere wirksame lokale und systemische Therapieoptionen zur Verfügung. Die Behandlung sollte inter- bzw. multidisziplinär geplant und durchgeführt werden.

Die Studienaktivität für Melanompatienten mit Hirnmetastasen hat erfreulicherweise deutlich zugenommen. Aktuell werden neue Therapieoptionen wie PD-1-Antikörper, Ipilimumab plus Nivolumab, BRAF-Inhibitoren plus MEKInhibitoren sowie stereotaktische Radiatio in Kombination mit Immuntherapie bzw. zielgerichteter Therapie untersucht.

\section{Fazit für die Praxis}

- Bei Verdacht auf Hirnmetastasen sollten ein craniales MRT und eine neurologische Untersuchung erfolgen.

- Bei der Therapieplanung sind prognostische Faktoren zu berücksichtigen.

- Die Therapie sollte interdisziplinär bzw. multimodal geplant und durchgeführt werden.

- Für singuläre Hirnmetastasen bietet sich die neurochirurgische Resektion oder stereotaktische Radiatio an.

- Bei 3 bis 4 Metastasen (ggf. $\leq 10$ Metastasen) und einer Größe von 0,5-4 cm kommt eine stereotaktische Radiatio in Betracht.

- Der Stellenwert der adjuvanten Ganzhirnradiatio ist nicht geklärt.
- Sowohl die BRAF-Inhibitoren Vemurafenib und Dabrafenib als auch der CTLA-4-Antikörper Ipilimumab haben in Studien eine intrakranielle Wirksamkeit gezeigt.

- Zerebrale Resistenzmechanismen scheinen die Wirksamkeit systemischer Therapien zu limitieren.

- Für multiple Hirnmetastasen stehen neben der Systemtherapie Ganzhirnbestrahlung und Supportivtherapie zur Verfügung.

- PD-1-Antikörper, Ipilimumab plus Nivolumab, BRAF-Inhibitoren plus MEK-Inhibitoren sowie stereotaktische Radiatio in Kombination mit Immuntherapie bzw. zielgerichteter Therapie werden in Studien untersucht.

\section{Korrespondenzadresse}

\section{Prof. Dr. F. Meier}

Hauttumorzentrum am Universitäts KrebsCentrum Dresden (UCC), Klinik und Poliklinik für Dermatologie, Universitätsklinikum CarlGustav-Carus Dresden, Technische Universität Dresden

Fetscherstr. 74, 01307 Dresden, Deutschland Friedegund.Meier@uniklinikum-dresden.de

Danksagung. Wir danken Dr. Maike Bergmann und Mario Rentsch für die Unterstützung bei der Textund Grafikformatierung.

\section{Einhaltung ethischer Richtlinien}

Interessenkonflikt. R. Rauschenberg erhielt Honorare für Beratungstätigkeiten von Novartis und GlaxoSmithKline; Teilnahmegebühren für Kongresse sowie Reisekostenübernahmen von Bristol-Myers Squibb. G. Tabatabai erhielt Honorare für Advisory Boards von MSD Schweiz, Roche Schweiz, BristolMyers Squibb; Reisekostenübernahmen von Roche Schweiz, MSD Schweiz, Medac; Unterstützung für präklinische Forschung von Medac und Roche Diagnostics. E.G.C. Troost gibt an, dass kein Interessenkonflikt besteht. M. Garzarolli erhielt Honorare für Beratungstätigkeiten von Roche, Bristol-Myers Squibb; Honorare für Vorträge von Roche Pharma, Bristol-Myers Squibb GlaxoSmithKline, Novartis, MSD; Reisekostenübernahmen von Roche Pharma, Bristol-Myers Squibb und AMGEN. S. Beissert erhielt Honorare für Beratungstätigkeiten und Reisekostenübernahmen von AMGEN. F. Meier erhielt Honorare für Beratungstätigkeiten von Roche, Bristol-Myers Squibb, GlaxoSmithKline, Novartis, MSD; Honorare für Vorträge von Roche Pharma, Bristol-Myers Squibb, GlaxoSmithKline, Novartis, MSD; Reisekostenübernahmen von Roche Pharma und Bristol-Myers Squibb; Unterstützung für präklinische Forschung von Wyeth/Pfizer, MerckSerono, Novartis; Unterstützung für klinische Forschung von Novartis.
Dieser Beitrag beinhaltet keine von den Autoren durchgeführten Studien an Menschen oder Tieren.

Open Access. This article is distributed under the terms of the Creative Commons Attribution 4.0 International License (http://creativecommons.org/ licenses/by/4.0/), which permits unrestricted use, distribution, and reproduction in any medium, provided you give appropriate credit to the original author(s) and the source, provide a link to the Creative Commons license, and indicate if changes were made.

\section{Literatur}

1. Ahmed KA, Freilich JM, Sloot S et al (2015) LINAC-based stereotactic radiosurgery to the brain with concurrent vemurafenib for melanoma metastases. JNeurooncol 122(1):121-126

2. Ajithkumar T, Parkinson C, Fife K et al (2015) Evolving treatment options for melanoma brain metastases. Lancet Oncol 16(13):e486-e497

3. Amer MH, Al-Sarraf M, Baker LH, Vaitkevicius VK (1978) Malignant melanoma and central nervous system metastases. Incidence, diagnosis, treatment and survival. Cancer 42(2):660-668

4. Aoyama H, Shirato H, Tago M et al (2006) Stereotactic radiosurgery plus whole-brain radiation therapy vs stereotactic radiosurgery alone for treatment of brain metastases: A randomized controlled trial. JAMA 295(21):2483-2491

5. Avril MF, Aamdal S, Grob JJ etal (2004) Fotemustine compared with dacarbazine in patients with disseminated malignant melanoma: A phase III study. JClin Oncol 22(6):1118-1125

6. Bernard ME, Wegner RE, Reineman K et al (2012) Linear accelerator based stereotactic radiosurgery for melanoma brain metastases. J Cancer Res Ther 8(2):215-221

7. Davies H, Bignell GR, Cox C et al (2002) Mutations of the BRAF gene in human cancer. Nature 417(6892):949-954

8. Giacomo AM Di, Ascierto PA, Queirolo P et al (2015) Three-year follow-up of advanced melanoma patients who received ipilimumab plus fotemustine in the Italian Network for Tumor Biotherapy (NIBIT)-M1 phase II study. Ann Oncol 26(4):798-803

9. Dummer R, Goldinger SM, Turtschi CP et al (2014) Vemurafenib in patients with BRAFV600 mutation-positive melanoma with symptomatic brain metastases: Final results of an open-label pilot study. Eur J Cancer 50(3):611-621

10. Eigentler TK, Figl A, Krex D et al (2011) Number of metastases, serum lactate dehydrogenase level, and type of treatment are prognostic factors in patients with brain metastases of malignant melanoma. Cancer 117(8):1697-1703

11. Fachinformation Ipilimumab. www.bmsonkologie.de. Zugegriffen: 31. Januar 2016

12. Fife KM, Colman MH, Stevens GN et al (2004) Determinants of outcome in melanoma patients with cerebral metastases. JClin Oncol 22(7):1293-1300

13. Fonkem E, Uhlmann EJ, Floyd SR et al (2012) Melanoma brain metastasis: Overview of current management and emerging targeted therapies. Expert Rev Neurother 12(10):1207-1215

14. Gaspar L, Scott C, Rotman M et al (1997) Recursive partitioning analysis (RPA) of prognostic factors in three Radiation Therapy Oncology Group (RTOG) brain metastases trials. Int J Radiat Oncol Biol Phys 37(4):745-751

15. Gaudy-Marqueste C, Carron R, Delsanti C (2014) On demand Gamma-Knife strategy can be safely combined with BRAF inhibitors for the treatment 
of melanoma brain metastases. Ann Oncol 25(10):2086-2091

16. Gaudy-Marqueste $C$, Regis JM, Muracciole $X$ et al (2006) Gamma-Knife radiosurgery in the management of melanoma patients with brain metastases: Aseries of 106 patients without wholebrain radiotherapy. Int J Radiat Oncol Biol Phys 65(3):809-816

17. Ghia A, Tomé WA, Thomas $S$ et al (2007) Distribution of brain metastases in relation to the hippocampus: Implications for neurocognitive functional preservation. Int J Radiat Oncol Biol Phys 68(4):971-977

18. Gondi V, Pugh SL, Tome WA et al (2014) Preservation of memory with conformal avoidance of the hippocampal neural stem-cell compartment during whole-brain radiotherapy for brain metastases (RTOG 0933): A phase II multi-institutional trial.J Clin Oncol 32(34):3810-3816

19. Goyal S, SilkAW, Tian Set al (2015) Clinical management of multiple melanoma brain metastases: $A$ systematic review. JAMA Oncol 1(5):668-676

20. Hecht M, Zimmer L, Loquai C et al (2015) Radiosensitization by BRAF inhibitor therapy -mechanism and frequency of toxicity in melanoma patients. Ann Oncol 26(6):1238-1244

21. Hodi FS, O'Day SJ, McDermott DF et al (2010) Improved survival with ipilimumab in patients with metastatic melanoma. N Engl J Med 363(8):711-723

22. Kaatsch $P$, Katalinic $A$, Hentschel $S$ et al (2012) Beiträge zur Gesundheitsberichterstattung des Bundes - Krebs in Deutschland 2007/2008, 8. Aufl. GEKID und Robert Koch-Institut, Berlin

23. Kefford RF, Maio M, Arance A et al (2013) Vemurafenib in metastatic melanoma patients with brain metastases: An open-label, singlearm, phase 2, multicenter study. Pigment Cell Melanoma Res 26:965

24. Kienast $Y$, Baumgarten $L$ von, Fuhrmann $M$ et al (2010) Real-time imaging reveals the single steps of brain metastasis formation. Nat Med 16(1):116-122

25. Kluger HM, Goldberg SB, Sznol Metal (2015) Safety and activity of pembrolizumab in melanoma patients with untreated brain metastases. J Clin Oncol 33:8035

26. Knisely JP, Yu JB, Flanigan J et al (2012) Radiosurgery for melanoma brain metastases in the ipilimumab era and the possibility of longer survival. J Neurosurg 117(2):227-233

27. Kohutek ZA, Yamada Y, Chan TA et al (2015) Long-term risk of radionecrosis and imaging changes after stereotactic radiosurgery for brain metastases. J Neurooncol 125(1):149-156

28. Larkin J, Ascierto PA, Dréno Betal (2014) Combined vemurafenib and cobimetinib in BRAF-mutated melanoma. NEngl J Med 371(20):1867-1876

29. Lawrence MS, Stojanov P, Polak P et al (2013) Mutational heterogeneity in cancer and the search for new cancer-associated genes. Nature 499(7457):214-218

30. Lee JH, Choi JW, Kim YS (2011) Frequencies of BRAF and NRAS mutations are different in histological types and sites of origin of cutaneous melanoma: A meta-analysis. Br JDermatol 164(4):776-784

31. Liebner DA, Walston SA, Cavaliere R et al (2014) Radiation necrosis mimicking rapid intracranial progression of melanoma metastasis in two patients treated with vemurafenib. Melanoma Res 24(2):172-176

32. Lin NU, Lee EQ, Aoyama $\mathrm{H}$ et al (2015) Response assessment criteria for brain metastases: Proposal from the RANO group. Lancet Oncol 16(6):e270-e278
33. Lin Q, Balasubramanian K, Fan D et al (2010) Reactive astrocytes protect melanoma cells from chemotherapy by sequestering intracellular calcium through gap junction communication channels. Neoplasia 12(9):748-754

34. Liniker E, Kong B, Menzies AM et al (2015) Safety and activity of combined radiotherapy and anti-PD-1 antibodies (anti-PD1) in patients with metastaticmelanoma. EurJCancer 1(suppl3):abstr 3302

35. Long GV, Stroyakovskiy D, Gogas H et al (2014) Combined BRAF and MEK inhibition versus BRAF inhibition alone in melanoma. $\mathrm{N}$ Engl J Med 371(20):1877-1888

36. Long GV, Trefzer U, Davies MA et al (2012) Dabrafenib in patients with Val600Glu or Val600Lys BRAF-mutant melanoma metastatic to the brain (BREAK-MB): A multicentre, open-label, phase 2 trial. Lancet Oncol 13(11):1087-1095

37. Ly D, Bagshaw HP, Anker CJ et al (2015) Local control after stereotactic radiosurgery for brain metastases in patients with melanoma with and without BRAF mutation and treatment. JNeurosurg 123(2):395-401

38. Manon R, O'Neill A, Knisely J et al (2005) Phasell trial of radiosurgery for one to three newly diagnosed brain metastases from renal cell carcinoma, melanoma, and sarcoma: An Eastern Cooperative Oncology Group study (E 6397). J Clin Oncol 23(34):8870-8876

39. Margolin K, Ernstoff MS, Hamid O et al (2012) Ipilimumab in patients with melanoma and brain metastases: An open-label, phase 2 trial. Lancet Oncol 13(5):459-465

40. Mathew M, Tam M, OttPA et al (2013) Ipilimumab in melanoma with limited brain metastases treated with stereotactic radiosurgery. Melanoma Res 23(3):191-195

41. Middleton MR, Grob JJ, Aaronson N et al (2000) Randomized phase III study of temozolomide versus dacarbazine in the treatment of patients with advanced metastatic malignant melanoma. JClin Oncol 18(1):158-166

42. MittapalliRK, Vaidhyanathan S, DudekAZ, Elmquist WF (2013) Mechanisms limiting distribution of the threonine-protein kinase B-RaFV600Einhibitor dabrafenib to the brain: Implications for the treatment of melanoma brain metastases. J Pharmacol ExpTher 344(3):655-664

43. Mittapalli RK, Vaidhyanathan $S$, Sane R, Elmquist WF (2012) Impact of P-glycoprotein (ABCB1) and breast cancer resistance protein $(A B C G 2)$ on the brain distribution of a novel BRAF inhibitor: Vemurafenib (PLX4032. J Pharmacol Exp Ther 342(1):33-40

44. Mornex F, Thomas L, Mohr P et al (2003) A prospective randomized multicentre phase III trial of fotemustine plus whole brain irradiation versus fotemustine alone in cerebral metastases of malignant melanoma. Melanoma Res 13(1):97-103

45. Narayana A, Mathew M, Tam M et al (2013) Vemurafenib and radiation therapy in melanoma brain metastases. J Neurooncol 113(3):411-416

46. Nayak L, Lee EQ, Wen PY (2012) Epidemiology of brain metastases. Curr Oncol Rep 14(1):48-54

47. Nazarian R, Shi H, Wang Q et al (2010) Melanomas acquire resistance to B-RAF (V600E) inhibition by RTK or N-RAS upregulation. Nature 468(7326):973-977

48. Niessner H, Forschner A, Klumpp B et al (2013) Targeting hyperactivation of the AKT survival pathway to overcome therapy resistance of melanoma brain metastases. Cancer Med 2(1):76-85
49. Okada H, Weller M, Huang R et al (2015) Immunotherapy response assessment in neurooncology: A report of the RANO working group. Lancet Oncol 16(15):e534-e542

50. Patchell RA, Tibbs PA, Regine WF et al (1998) Postoperative radiotherapy in the treatment of single metastases to the brain: A randomized trial. JAMA 280(17):1485-1489

51. Patel PM, Suciu S, Mortier L et al (2011) Extended schedule, escalated dose temozolomide versus dacarbazine in stage IV melanoma: Final results of a randomised phase III study (EORTC 18032). Eur J Cancer 47(10):1476-1483

52. Peuvrel L, Ruellan AL, Thillays F et al (2013) Severe radiotherapy-induced extracutaneous toxicity under vemurafenib. Eur J Dermatol 23(6):879-881

53. Peuvrel L, Saint-Jean M, Quéreux G et al (2014) Incidence and characteristics of melanoma brain metastases developing during treatment with vemurafenib. J Neurooncol 120(1):147-154

54. Pflugfelder A, Kochs C, Blum A et al (2013) Malignant Melanoma S3-Guideline "Diagnosis, Therapy and Follow-up of Melanoma“. J Dtsch Dermatol Ges 11(s6):1-116

55. Raizer JJ, Hwu WJ, Panageas KS et al (2008) Brain and leptomeningeal metastases from cutaneous melanoma: Survival outcomes based on clinical features. Neuro Oncol 10(2):199-207

56. Rauschenberg R, Garzarolli M, Dietrich U et al (2015) Systemic therapy of metastatic melanoma. JDtsch Dermatol Ges 13(12):1223-1237

57. Rompoti N, Schilling B, Livingstone $E$ et al (2013) Combination of BRAF inhibitors and brain radiotherapy in patients with metastatic melanoma shows minimal acute toxicity. J Clin Oncol 31(30):3844-3845

58. S2k-Leitlinie 030/060: Hirnmetastasen und Meningeosis neoplastica-Stand:03/2014

59. Schild SE, Behl D, Markovic SN et al (2010) Brain metastases from melanoma: Is there a role for concurrent temozolomide in addition to whole brain radiation therapy? Am J Clin Oncol 33(6):633-636

60. Silk AW, Bassetti MF, West BT et al (2013) Ipilimumab and radiation therapy for melanoma brain metastases. Cancer Med 2(6):899-906

61. Solit DB, Rosen N (2011) Resistance to BRAF inhibition in melanomas. N Engl J Med 364(8):772-774

62. Soon YY, Tham IWK, Lim KH et al (2014) Surgery or radiosurgery plus whole brain radiotherapy versus surgery or radiosurgery alone for brain metastases. Cochrane Libr 3:CD009454

63. Staudt M, Lasithiotakis K, Leiter U et al (2010) Determinants of survival in patients with brain metastases from cutaneous melanoma. Br J Cancer 102(8):1213-1218

64. Strik H (2015) Individuell angepasste Diagnostik und Therapie. Focus Onkol 18:44-51

65. Wilson EH, Weninger W, Hunter CA (2010) Trafficking of immune cells in the central nervous system. J Clin Invest 120(5):1368-1379

66. Yamamoto M, Serizawa T, Shuto T et al (2014) Stereotactic radiosurgery for patients with multiple brain metastases (JLGK0901): A multiinstitutional prospective observational study. Lancet Oncol 15(4):387-395

67. Zahnreich S, Mayer A, Loquai C et al (2016) Radiotherapy with BRAF inhibitor therapy for melanoma: Progress and possibilities. Future Oncol 12(1):95-106

68. Zindler JD, Thomas CR, Hahn SM et al (2016) Increasing the therapeutic ratio of stereotactic ablative radiotherapy by individualized isotoxic dose prescription. J Natl Cancer Inst 108(2):djv305 